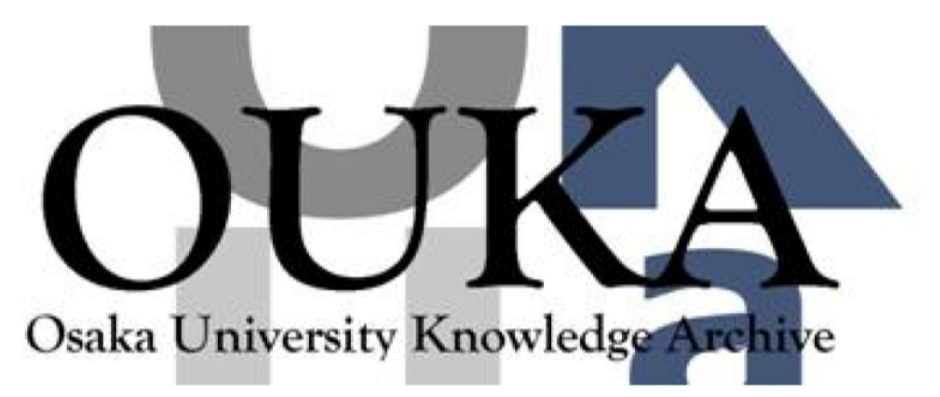

\begin{tabular}{|c|l|}
\hline Title & $\begin{array}{l}\text { Development of x-ray emission computed } \\
\text { tomography for ICF research }\end{array}$ \\
\hline Author(s) & Nakai, M.; Shinohara, S.; Katayama, M. et al. \\
\hline Citation & $\begin{array}{l}\text { Review of Scientific Instruments. 61(10) } \\
\text { p. 2783-p. 2785 }\end{array}$ \\
\hline Issue Date & $1990-10$ \\
\hline oaire:version & VoR \\
\hline URL & https://hdl. handle. net/11094/3304 \\
\hline rights & \\
\hline Note & \\
\hline
\end{tabular}

Osaka University Knowledge Archive : OUKA

https://ir. Library. osaka-u. ac. jp/

Osaka University 


\title{
Development of $x$ aray emission computed tomography for ICF research
}

\author{
M. Nakai, S. Shinohara, M. Katayama, Y.W. Chen, S. Kobayashi, N. Miyanaga, \\ K. A. Tanaka, K. Nishihara, M. Yamanaka, T. Yamanaka, and S. Nakai \\ Institute of Laser Engineering, Osaka University, Suita Osaka 565, Japan
}

(Presented on 7 May 1990)

\begin{abstract}
A computed tomographic (CT) technique has been developed to diagnose laser-irradiated spherical targets using $x$-ray emissions. The three-dimensional (3-D) $x$-ray distribution was reconstructed by using an algebraic reconstruction technique (ART) from $x$-ray pinhole camera images obtained along different sight directions. 3-D distributions of electron temperature and density were measured by use of an absorption filter technique. Time-resoived 3-D x-ray emission images from an imploding hollow shell target were reconstructed with less than $100 \mathrm{ps}$ temporal resolution by using $\mathrm{x}$-ray multiframing cameras.
\end{abstract}

\section{INTRODUCTION}

It has been recognized that the compression of the hquid fuel (deuterium and/or tritium) up to 500-1000 times the liquid density is necessary to ignite thermonuclear burn in an inertial confinement pellet. Implosion uniformity is a key issue for achievement of such a high compression. Shapes of the imploding pellet and compressed core plasma have been observed by $\mathrm{x}$-ray and particle imaging diagnostics. ${ }^{1,2}$ But a single image could only give two-dimensional information integrated along a particular direction. In order to evaluate the three-dimensional nonuniformity, a tomographic technique is desired. We have developed a computed tomographic (CT) technique to reconstruct the three-dimensional $x$-ray emission distribution from several $x$-ray pictures taken by $x$-ray pinhole cameras from different directions.

The implosion process is completed in a very short time, typically 1 ns. Therefore it is impossible to use a moving detector, as is used in the medical research, to spatially scan the object. In implosion experiments many kinds of detectors are attached to the vacuum chamber, in the center of which the pellet target is irradiated by energy drivers. All the required data must be acquired simultaneously in a single shot using a limited number of detectors. Thus, measurement of the 3-D $\mathrm{x}$-ray emission distribution is a reconstruction problem from incomplete projection data with some a priori constraints. We have adopted an algebraic reconstruction technique as an algorithm because it can manage a large matrix of data and incorporate a priori knowledge into the reconstruction procedure. ${ }^{3.6}$ Spectral information can be used to determine plasma parameters. Here we use information obtained from a set of filtered images to obtain the 3-D distributions of electron temperature and relative density.

In order to perform the computed tomography, it is necessary to develop accurate detectors with rapid data analysis capability. When nonlinear functions are included in the projection measurements, various distorting artifacts having distinctive shapes are produced through reconstruction processes. 7,8 Therefore we designed two types of $x$-ray image detectors with CCD (charge coupled device) cameras. ${ }^{9,10}$ The first is just a phosphor, which provides timeintegrated signals but has constant spectral sensitivity suitable for the absorption filter method. The other is an $x$-ray framing camera, which provides temporal resolution better than $100 \mathrm{ps}$. Using the CCD cameras as image detectors, the direct acquisition system can be constructed.

In this paper, after the description of the $x$-ray $C T$ system on the Gekko XII facility at ILE Osaka, we present examples reconstructing the 3-D temperature distribution.

\section{SYSTEM CONSTRUCTION}

The $x$-ray CT system for implosion experiments at ILE consists of pinhole adjusters, $\mathrm{x}$-ray imaging detectors, $\mathrm{CCD}$ camera sets, and frame memories (Fig. 1 ). Each twodimensional image is accumulated from the $\mathrm{CCD}$ in a frame memory, then transferred to the main computer (NEC-ACOS). The set of two-dimensional images is then reconstructed to obtain a $3-\mathrm{D}$ image. Each $\mathrm{x}$-ray imaging detector is coupled to a thermoelectrically cooled $C C D$ camera through relay lens optics $(F / 2)$. The CCDs and frame mernories are commercially availabie (Hamamatsu, $\mathrm{C} 3140$ and $\mathrm{C} 3610$ ). The $\mathrm{CCD}$ has a $510 \times 492$ pixel format in an area of $8.6 \times 6.6 \mathrm{~mm}^{2}$. They are used at a frame rate of $1 \mathrm{~Hz}$, signal charge is accumulated on each pixel for $1 \mathrm{~s}$, then digitized by a 12 bit $\mathrm{A} / \mathrm{D}$ converter. The rms noise levels are below 50 electrons/pixel at $-30^{\circ} \mathrm{C}$ and specified dynamic range is more than 2000.

Two types of $x$-ray image converters are used. The first is an optical fat overcoated with $B 20$ phosphor $[(\mathrm{Zn}, \mathrm{Cd}) \mathrm{S}: \mathrm{Ag}]$ of $10 \mu \mathrm{m}$ in thickness. Grain size of the phosphor was about $2 \mu \mathrm{m}$. Magnification of the relay optics was designed to be $1 / 2$ and the effective area on the phosphor was $17 \times 13 \mathrm{~mm}^{2}$. The second type of detector is a multiframe framing camera. ${ }^{13}$ This is a gated microchannel plate (MCP) image converter, which consists of a pair

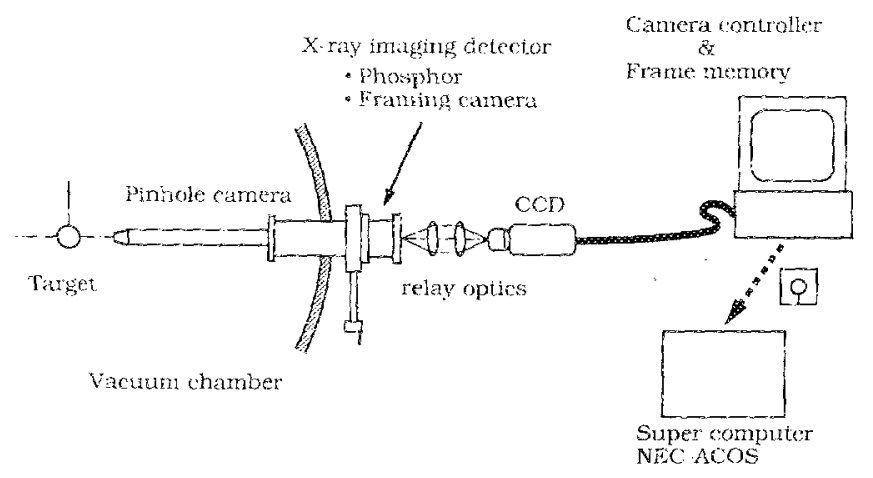

FIG. 1. Schematic diayram of the $\mathrm{x}$-ray $\mathrm{Cr}$ system on Gekko XII. 
of MCPs and a phosphor screen. The first MCP is used as a gated photocathode with its electrode in a strip line configuration gated by an electric pulse; the second MCP is an electron multiplier as usual. Images are formed along the strip line by a pinhole array at the time when the electric pulse passes through each point. Thus we can obtain six sequential temporally resolved images with a time interval of 100 ps. According to the experimental resuit using the 4th harmonic of a glass laser with a puise duration of about $10 \mathrm{ps}$, the temporal resolution was about $80 \mathrm{ps}$ and the dynamic range was more than $10^{2}$ with linearity within an error of $\pm 20 \%$. The spatial resolution was evaluated to be $101 \mathrm{p} / \mathrm{mm}$ (at $\mathrm{MTF}=20 \%$ ) by measuring the modulation transfer function.

Reconstruction of 3-D x-ray emission distribution uses an inversion technique to estimate a $3 \mathrm{D}$ distribution $I(x, y, z)$ from its several $2 \mathrm{D}$ projections. Since the visual angle of each pinhole camera is very small, we can regard our system as in parallel geometry. Assuming that the selfabsorption of $x$ rays within the plasma in question is negligible, obtained images are regarded as parallel projections along the viewing directions. In order to determine the three-dimensional distribution $I(x, y, z)$ from such twodimensional projections obtained at different viewing directions, an iterative method known as the multiplicative algebraic reconstruction technique was used. ${ }^{2}$

The major advantage of ART is that a priori knowledge can be incorporated into the reconstruction procedure. ${ }^{4}$ As the constraints, we incorporated a positivity and boundary condition; the $k$ th reconstruction $I^{k}$ should be zero outside the initial target. Namely, in each iteration negative $I^{k_{S}}$ and $I^{k_{S}}$ outside the initial target radius were set to be zero.

In order to evaluate the accuracy of the reconstruction, computer simulations were performed using the phantom shown in Fig. 2 (a). The phantom contains two spheres of relative intensities 1,3 and relative radii 24,9 , respectively. The central intense sphere [shaded area in Fig. 2(a)] has been truncated and shifted by three pixels from the center along for each $x, y$, and $z$ axis respectively on purpose. The averaged intensity error $\delta I / I$ (rms) and the averaged deviation of the size of center core [shaded area in Fig. 2(a)] $\delta R / R$ between the original object and reconstructed image were calculated [Fig. 2(b)]. The dashed lines and solid lines show the accuracy of reconstruction with and without addition of Poisson noise to each projection, where the signal-to-noise ratio was assumed to be 10 . As seen in the figure, the accuracy of reconstruction is improved by increasing the number of detectors, the deviations $\delta I / I$ and $\delta R / R$ are estimated to be $\sim 30 \%$ and $\sim 16 \%$ with three detectors. Another measure of the reconstruction accuracy is shown in Fig. 3. Spread of a point source in FWHM is shown as the spatial resolution in the figure. The solid square is an experimental value obtained from the spread of the surface emission of a plastic shell target $(800 \mu \mathrm{m}$ in diameter) irradiated by short-pulse (100 ps FWHM) laser beams. The spatial resolution of about $40 \mu \mathrm{m}$ was provided using three pinhole cameras.
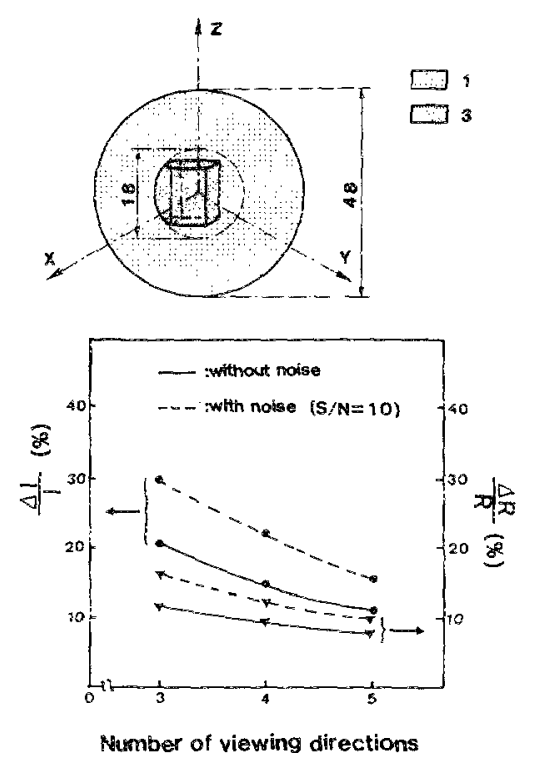

FIG. 2. Reconstruction accuracy was evaluated by computer simulation using a phantom (a). The intensity error $(\delta / / I)$ and the core size deviation $(\delta R / R)$ between the reconstructed image and original object are improved with increasing the number of viewing directions.

If the absorption opacity of the object plasma can be neglected, the emission coefficient at each point is derived by the method described above. Knowing the spectral emission coefficient we can calculate the temperature and density of the plasma. When the x-ray photon energy $h v$ in the observed region is higher than the ionization energy of the plasma, the dominant radiation process is bremsstraklung. In such a situation one can obtain the electron temperature of the plasma by the absorption filter technique. Assuming a Maxwellian electron-energy distribution, the spectral emission coefficient due to bremsstrahlung is described as a function of electron density $n_{e}$ and temperature $T_{e}$. The three-dimensional distribution $I(x, y, z)$ reconstructed from the projections taken by the detector with the spectral sensitivity $S(v)$ through an absorption filter of the thickness $l$ is

$$
\begin{aligned}
I(x, y, z) \propto & \frac{z}{k T_{e}(x, y, z)} n_{e}(x, y, z)^{2} \exp \left(-\frac{h v}{k T_{e}(x, y, z)}\right) \\
& \times \exp [-\mu(h v) l] S(v) d v,
\end{aligned}
$$

where $\mu(h v)$ is a mass absorption coefficient of the filter material. Therefore the ratios $R(x, y, z) s$ among $I(x, y, z) s$

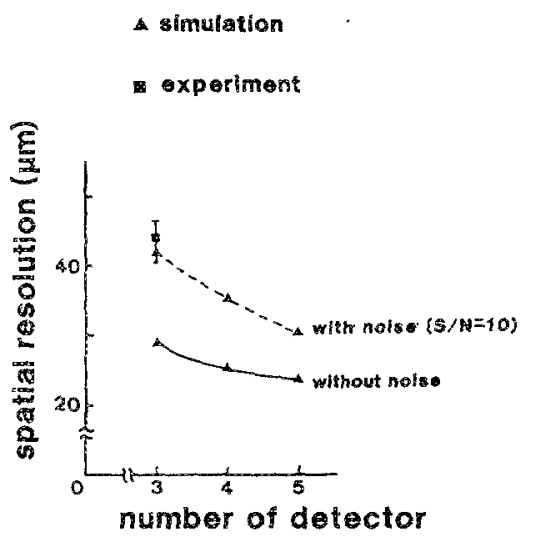

FIG. 3. Full wioth of the half maximum of the reconstructed image of a point emission was calculated varying the number of the viewing directions, as the measure of the spatial resolution. 


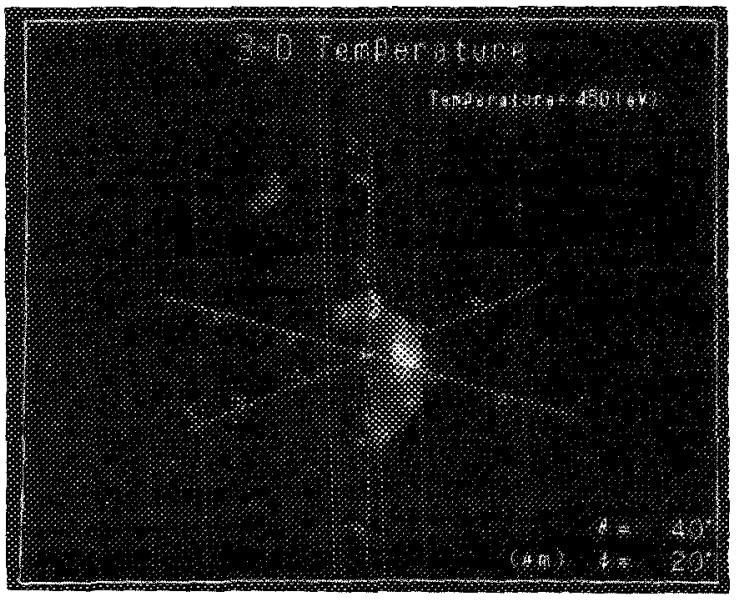

FIG. 4. 3-D display of the $450 \mathrm{eV}$ isotemperature surface in the imploded core of a cryogenic foam shell target.

obtained from the projections using absorption filters of different thicknesses are not dependent on $n_{e}$ but a function of $T_{e}$. Thus one can obtain the temperature $T_{e}(x, y, z)$ from the ratio $R(x, y, z)$. The relative density distribution can then be obtained from Eq. (1). It is very difficult to calculate the density distribution directly because it is difficult to absolutely calibrate the detectors. The absolute value could be deduced by knowing the residual mass or densityradius product from other measurements.

\section{HI. APPLICATIONS}

In order to measure the time-integrated threedimensional temperature distribution in the imploded core plasma of a cryogenic target, ${ }^{12,13}$ images were obtained from three sets of the phosphor coupled with the CCD cameras. Four pinhole images through aluminum filters with different thicknesses of $2,3,6,10 \mu \mathrm{m}$ were arranged on each phosphor. The diameter of each pinhole was normally $20 \mu \mathrm{m}$ and the magnification of pinhole camera was 8. The target was a foam shell with an outside diameter of $780 \mu \mathrm{m}$ and a wall thickness of $35 \mu \mathrm{m}$ filled with liquid deuterium. Twelve laser beams from a frequency-doubled Gekko XII glass laser system were focused onto the target with a puise duration of 1 ns and a total energy of $8 \mathrm{~kJ}$.

Figure 4 shows the isotemperature surface at $450 \mathrm{keV}$ in the core. In the present status of the cryogenic experiments, it is very difficult to fill the foam shells with deuterum completely and unirormly; the resuitant cores are severely distorted in some cases. Although the 2-D x-ray image was somewhat round and more uniform, the reconstructed temperature was shown to have a nonuniform distribution as in Fig. 5. In this shot, the core temperature ranged from 200 to $1000 \mathrm{eV}$. Since the inferred temperature is proportional to the intensity ratio $R$ to the power of 1.7 , the error in the calculated temperature is estimated to be $30 \%$ due to the reconstruction error in the intensity distribution.

The experimentally measured areal density $\rho R$ of the compressed core of the cryogenic foam shell was $<10 \mathrm{mg} /$ $\mathrm{cm}^{2}$ and the temperature was much higher than the ionization energy of carbon (which has the highest $\mathbb{Z}$ number in the target). Therefore all the assumptions we described previously were fulfilled.

The reconstruction of the temporally resolved 3-D xray image was demonstrated using two sets of the identical $\mathrm{x}$-ray framing cameras. Each framing camera was arranged to provide six sequential images with a time interval of 100 ps. The timing jitter of two sets of the x-ray framing cameras was measured by monitoring driving electric pulses on a $6 \mathrm{GHz}$ digitizing oscilloscope (Tektronix 7250). Practically two sets of detectors are not sufficient to perform the tomographic reconstruction. $\langle\delta I / I\rangle$ was calculated to be larger than $50 \%$ using a phantom simulating a spherical shell piasma. We defer detailed analysis until we have finished the installation of three sets of the X-ray framing cameras and we fully understand reconstruction artifacts on the distinctive shapes. In order to discuss the evolution of the deformation of the shell and corresponding nonuniformity of the compressed core, it is necessary to expand the distribution to a proper set of orthogonal functions.

\section{SUNMARY}

The x-ray ernission $C T$ systern has been constructed using phosphor $(\mathrm{Zn}, \mathrm{Cd}) \mathrm{S}: \mathrm{Ag}$ as a time-integrated detector and an $\mathrm{X}$-ray framing camera as a temporally resolved detector in order to investigate the three-dimensional nonuniformity of the imploding pellet target in an inertial confinement fusion experiment. Reconstruction accuracy was evaluated by computer simulation using a phantom of a compressed core. Deviation in intensity and spatial resolution were estimated to be $20 \%$ and $40 \mu \mathrm{m}$, respectively, in the case of using three detectors. 3-D distributions of electron temperature and density were reconstructed using an absorption filter technique. We successfully applied this system to measure implosions of cryogenic targets. In order to study the 3-D evolution of the target nonuniformity, it is essential to expand the distribution of the physical parameter to a proper set of orthogonal functions. From this point of view the reconstruction accuracy must be evaluated.

${ }^{1}$ N. M. Ceglio and L. W. Coleman, Phys. Rev. Lett. 39, 20 (1977).

${ }^{2}$ Y. W. Chen, N. Miyanaja, M. Yamanaka, H. Azechi, K. Nishihara, Y. Kato, T. Yamanaka, and S. Nakai, J. Appl. Phys. (to be published). ${ }^{3}$ G. T. Herman, A. Lent, and S. W. Rowland, 3. Theor. Biol. 42, 1 (1973).

${ }^{4}$ R. Rangayyan, A. P. Dhawan, and R. Gordon, Appl. Opt. 24, 4000 (1985).

${ }^{5}$ M. R. Civanlar and F. J. Trussell, ICASSP 86, Tokyo (1986).

'Y. W. Chen, N. Miyanaga, M. Yamanaka, M. Nakai, K. A. Tanaka, K. Nishihara, T. Yamanaka, and \$. Nakai, J. Appl. Phys. (to be published).

A. J. Duerinckx and A. Macovski, iEEE Trans. Nucl. Sci. NS-26, 2848 (1979).

${ }^{8}$ A. Macovski, Proc. IEEE 71, 373 (1983).

${ }^{4}$ I. H. Kinney, Q. C. Johnson, U. Bonse, R. Nusshardt, and M. C. Nichols, SPIE 691, 43 (1986).

${ }^{10}$ S. Gruner, Rev. Sci. Instrum. 60, 1545 (1989).

${ }^{11}$ M. Katayama, M. Nakai et al., Proc. of European Conf. on Optics, 24-28 April, Paris, 1989. This camera has been developed in ILE and manufactured at Hamamatsu Photo, $\mathrm{K}$. K.

${ }^{12}$ T. Norimatsu et al., J. Vac. Sci. Technol. A 6, 3144 (1987).

${ }^{13} \mathrm{~T}$. Yamanaka et al, in Laser Interaction with Matter, edited by $G$. Velarde, E. Minguez, and J. M. Perlado (World Scientific, Teaneck, NJ, 1989).

${ }^{14} \mathrm{Y}$. Kato et al, ILE Quarterly Progress Report, ILE-QPR-84-8. 